\title{
Low Light Underwater Image Enhancement Algorithm Based on Fusion Histogram Equalization Technique
}

\author{
Rafid A. Haleot \\ Department of computer \\ College of Education \\ Mustansiriyah University \\ Baghdad- Iraq
}

\begin{abstract}
One of the main challenges during monitoring the underwater images is unbalanced illumination and poor contrast. These problems added a hazy appearance in the visuals for underwater images. Effective underwater image enhancement fusion algorithm founded on CLAHE technique for deferent color spaces were used in this article. At first, convert the original underwater image from RGB space to HSV space. Then, the algorithm separately applies CLAHE in this color space. After that, the HSV enhanced images are converted back to RGB spaces. This enhanced image convert to LAB space and sharpening filter is applied to demonstrate the contrast and converted back to RGB spaces. The results of investigational work show that, contrasted and the customary enhancement methods of underwater images, such that MSE, UQI and PSNR, of the proposed strategy are joined ideally. And has been seen that proposed framework superior to previously existing strategies for improvement of underwater image. It can be seen that the details proposed method of image that more abundant, image brightness is improved, and color is more distinct and the image details are clearer.
\end{abstract}

Keywords: Underwater Images, CLAHE, Color Spaces, Sharpening Filer.

\section{1- INTRODUCTION}

Besides common images, underwater image suffers from poor visibility, poor clarity, low contrast, low resolution, uneven illumination and low signal-to-noise ratio because of the attenuation process of the propagated light. Light lessening and backscattering genuinely fall apart the image in the imaging procedure. Refraction and furthermore ingestion by water are liable for changes in shading and pale blue tone in the image [1,2]. Each shading ingests at an alternate rate on the grounds that each shading has an alternate frequency and vitality level. The blue light has the briefest frequency, so it can spread far and wide underwater. The factor of profundity likewise has an influence here. As we go further and more profound under the oceans the hues with bigger frequencies are assimilated more. Light power will rot significantly more rapidly in the water, particularly in profound water making a somewhat blue tone the image, and the scene is portrayed by helpless difference [3]. To improve the nature of such images and scenes, the image upgrade should be finished. The honing strategies for the underwater image can be partitioned into two techniques: image reclamation strategy and image improvement strategy. Image upgrade is a strategy used to improve the optical nature of images to guarantee the better visual quality or better extraction of data from the image. The "underwater image" improvement strategy doesn't consider the imaging instrument of the image, yet just spotlights on the best way to accomplish the image upgrade impact by modifying the pixel estimation of the image. Histogram balance [4] is a strategy such image upgrade procedure that used to improved underwater images because of its capacity to upgrade differentiate. The standard method for this situation is to re-map dim sizes of the image with the goal that the resultant histogram approximates that of uniform appropriation [5, 6]. The work presented in this paper aims to develop new tool for improving underwater images, The input images are converted in HSV color space and applied CLAHE algorithm and then applying sharpening filter only on the luminance (L) channel in the Lab space. After remapping to the RGB space and obtained the input image enhanced version. The paper organized as: in the following section we presented the related work consisting of different techniques. Section 3 introduce the method used for enhancement including (CLAHE) .Section 4 describe the color models used in this paper. Section 5 discusses the proposed method. Section 6 presents the results and discus the proposed work .section 6 present the statistical matrices that used for quality measure. Section 7 discuse the results and future work. 


\section{2- RELATED WORK}

The several algorithms were working to enhance the quality of "underwater images" by various methodologies, Zou et al [7] aimed to color correct of "underwater image" by brightness improve through white balance improved algorithm based on Laplacian fusion. Raj et al, [8] used adaptive histogram equalization which is a trade-off between worldwide histogram balance and completely versatile histogram evening out-local histogram, the recently acquired upgraded variant of the info image is gotten. Qiao, et al [9] introduced a technique dependent on (CLAHE-WT) where WT is wavelet transform, to upgrade the ocean cucumber quality of image, while Luo, et al [10] attempted to take care of the issue of the lopsided enlightenment and clamor in underwater images Contrast Limited utilizing "Adaptive Histogram Equalization" innovation spatial area to upgrade the Adaptive of various locales of the image contingent upon the conveyance of pixel estimations of every district. At that point utilized Holomorphic Filtering innovation to improve the image subtleties and decrease the commotion in the recurrence space. Mangeruga et al. [11] chosen a few calculations from the cutting edge and utilized them to improve a dataset of images created in different underwater sites. Garg et.al. [12] have utilized mixing of CLAHE and percentile procedures, they saw that their proposed strategy performs great outcome contrasting and another existed methods for underwater image upgrade. Zheng et al. [13] portrayed a technique which it works just on single debased "underwater image". They endeavored to portray CLAHE change and USM changes for image upgrade. They have additionally referenced that Hue varieties are constrained by frequency pay and underwater images have decreasing shades, low perceptibility, and little dissimilarity.

\section{3- HISTOGRAM EQUALIZATION}

This method used contrast modifying of image, and evaluation the intensities image depending on histogram. The available gray levels probability distribution is key of method to gray levels mapping. The digital image histogram has range gray levels with [0, L-1] defined as the "frequency distribution function" overall intensity distribution of the image.

$$
\mathrm{H}\left(\mathrm{X}_{\mathrm{r}}\right)=\mathrm{n}_{\mathrm{r}}
$$

where $X_{r}$ is the $r^{\text {th }}$ input (gray level) image of, and $n_{r}$ is the pixels number in image with gray level $X_{r} . r=0,1,2 \ldots L-1$, The Probability Density Function (PDF). Can be define as

$$
\mathrm{P}\left(\mathrm{X}_{\mathrm{r}}\right)=\mathrm{n}_{\mathrm{r}} / \mathrm{n}
$$

$\mathrm{n}$ : Pixels total number in the image. The "Cumulative Density Function" defines as:

$$
\operatorname{CDF}\left(\mathrm{X}_{\mathrm{r}}\right)=\Sigma_{\mathrm{i}=0}^{\mathrm{k}} \mathrm{P}\left(\mathrm{X}_{\mathrm{i}}\right)=\Sigma_{\mathrm{i}=0}^{\mathrm{k}} \mathrm{n}_{\mathrm{i}} / \mathrm{n}
$$

HE: Scheme maps of input image with range $\left[\mathrm{X}_{\mathrm{o}}, . ., \mathrm{X}_{\mathrm{L}-1}\right]$, by using :

$$
\mathrm{T}\left(\mathrm{X}_{\mathrm{r}}\right)=\mathrm{X}_{\mathrm{L}-1} \times \operatorname{CDF}\left(\mathrm{X}_{\mathrm{r}}\right)
$$

where $T\left(X_{r}\right)$ is transfer function, $X_{L-1}$ is max value of gray level

The disadvantage in the Histogram Equalization Method is the decrease background noise of amplification in the dynamic signal. AHE beats the restrictions of common histogram evening out by make change of every pixel with a specific change work got from a local area. One of the downsides of versatile histogram balance is that it tends to over intensify commotion in moderately homogeneous locales of image [14]. This restriction can be overwhelmed by applying a variety of versatile histogram balance called (CLAHE), which is a derivation of the Adaptive histogram equalization. By using (CLAHE) the contrast of the image is remain constant and histogram equalization is done. CLAHE was d intended to forestall the over-enhancement of clamor that can be created by utilizing the versatile histogram leveling. The image is apportioned into tiles which can be depicted as little areas in the image that separated in understanding to a specific matrix to misuse nearby spatial lucidness. The difference of each tile is upgraded by apply the histogram evening out and forestalls any expansion of commotion to image particularly in homogeneous territories of the image [15].

\section{4- COLOR SPACES}

In digital image, RGB color space is consists independent images was three components (red (R), green $(\mathrm{G})$, and blue (B)). The HSV color space is intended from the prime RGB color space by nonlinear transformation. HSV color space [14] is based on human visual observation theory and is appropriate for deciphering and portraying shading. So it is a lot more like a people impression of shading, this model depicts the shading space as far as chromatic information: hue (H), saturation (S), and achromatic information: value (V) components. The force part is the weighted normal of three shading channels and less touchy to noise. HSV color space was best frequently has use application-oriented space.

$$
\begin{aligned}
& \mathrm{S}=\frac{\max (\mathrm{R}, \mathrm{G}, \mathrm{B})-\min (\mathrm{R}, \mathrm{G}, \mathrm{B})}{\max (\mathrm{R}, \mathrm{G}, \mathrm{B})} \\
& \mathrm{V}=\max (\mathrm{R}, \mathrm{G}, \mathrm{B}) .
\end{aligned}
$$


Lab color space is molded with L (Lightness cannel) and (a, b) (chromatic segments). This shading space can communicate a more extensive shading extent or range than RGB and HSV because it includes all perceivable colors and that means its gamut exceeds than RGB color model [15]. One of the most important attributes of the Lab color space is the device independency, so is used as an interchange format between different devices [16].

$$
\begin{aligned}
& L^{*}=116 f\left(\frac{Y}{Y_{n}}\right)-16 \\
& a^{*}=1500\left(f\left(\frac{X}{X_{n}}\right)-f\left(\frac{Y}{Y_{n}}\right)\right) \\
& b^{*}=200\left(f\left(\frac{Y}{Y_{n}}\right)-f\left(\frac{Z}{Z_{n}}\right)\right)
\end{aligned}
$$

\section{5- PROPOSED ALGORITHM}

The Proposed algorithm is explained in figure (1) that shows how obtain more enhanced underwater image. All images are randomly chosen from the internet in RGB format image, our specific algorithm process for underwater image enhancement is: First, Image (RGB space) convert to HSV space. Second, CLAHE applied to the intensity component (v) in the HSV color space to enhance the brightness information contrast, while the chromatic information is preserve, Then, the enhanced HSV color space image transformed back to RGB color space. After enhancing the contrast of the images, we further convert the images to L*a*b* space and sharpening apply on $\mathrm{L}^{*}$ channel. The reason for doing this is mainly to get an improved illuminance component and better contrast underwater images. Finally image back to RGB space, and fusion done by combined the enhanced image in the HSV space with the last enhance image using image ADD function to achieve optimal enhancement.

\section{6- QUANTITATIVE STATISTICAL}

There are many Quantitative statistics, by calculating which the improved image quality is evaluated, are Calculated were for the evaluation algorithms quality of the enhanced underwater images.

- Riesz-transform based Feature Similarity (RFSIM) index: This scale is based on Novel Feature based on a model to evaluate the image quality (IQA) and Riesz Transforms based on the similarity feature scale that was proposed on the basis of the fact that the human vision system (HVS) mainly perceives the image according to its low-level properties. Calculate (RFSIM) from the following equation:

$$
\operatorname{RFSIM}=\prod_{i=1}^{5} \frac{\sum \sum \frac{2 \mathrm{x}(\mathrm{i}, \mathrm{j}) \cdot \mathrm{y}(\mathrm{i}, \mathrm{j})+\mathrm{c}}{\mathrm{x}^{2}(\mathrm{i}, \mathrm{j})+\mathrm{y}^{2}(\mathrm{i}, \mathrm{j})+\mathrm{c}} \cdot \mathrm{M}(\mathrm{x}, \mathrm{y})}{\sum \sum \mathrm{M}(\mathrm{x}, \mathrm{y})}
$$

Where $\mathrm{M}(\mathrm{x}, \mathrm{y})$ is the location property mask $(\mathrm{i}, \mathrm{j})$ and $\mathrm{c}$ is a constant whose value is small.

- Root Mean Square Error (RMSE): This measure assesses the deviation sum present at intertwined image contrasting and reference. RMSE has been defining as:

$$
\text { RMSE }=\sqrt{\frac{1}{M N} \sum_{i=1}^{M} \sum_{j=1}^{N}(x(i, j)-y(i, j))^{2}}
$$

The quality of the image is better if the RMSE value is to be as low as possible.

- Peak Mean Square Error (PMSE): has been defining as:

$$
\operatorname{PSME}=\frac{1}{\mathrm{MN}} \times \frac{\sum_{\mathrm{i}=1}^{\mathrm{M}} \sum_{\mathrm{j}=1}^{\mathrm{N}}[\mathrm{x}(\mathrm{i}, \mathrm{j})-\mathrm{y}(\mathrm{i}, \mathrm{j})]^{2}}{[\operatorname{MAX}(\mathrm{x}(\mathrm{i}, \mathrm{j}))]}
$$

- Laplacian Mean Square Error (LMSE): This scale depends on the significance of edge detection. The largest value (LMSE) between the two images indicates that the image possesses low Quality. This scale is defined:

$$
\operatorname{LMSE}=\frac{\sum_{\mathrm{i}=1}^{\mathrm{M}} \sum_{\mathrm{j=1}}^{\mathrm{N}}\left[\mathrm { L } \left(\mathrm{x}(\mathrm{i}, \mathrm{j})-\mathrm{L}\left(\mathrm{y}(\mathrm{i}, \mathrm{j}]^{2}\right.\right.\right.}{\sum_{\mathrm{i}=1}^{\mathrm{M}} \sum_{\mathrm{j}=1}^{\mathrm{N}}\left[\mathrm{L}(\mathrm{x}(\mathrm{i}, \mathrm{j})]^{2}\right.}
$$


- Peak Signal to Noise Ratio (PSNR): This assesses PSNR between two images. This proportion is now and then used quantify of quality between a unique image and a reproduced image. PSNR is a measure for the pinnacle blunder coming about because of the setting metric of (MSE) for greatest conceivable luminance esteem and characterized as:

$$
\text { PSNR }=10 * \log _{10}\left(\frac{\mathrm{R}^{2}}{\mathrm{MSE}}\right)
$$

Where R: Gray levels number and their range is (0-255) and describes the maximum variance at the data type of input image, for example, the value $\mathrm{R}=255$ if the data type of input image is (8-bit).

- Mean Square Error (MSE): is large value means quality of image is poor. The definition of MAE is:

$$
\text { MAE }=\frac{1}{M N} \sum_{i=1}^{M} \sum_{j=1}^{N}|x(i, j)-y(i, j)|
$$

- Correlation Quality (CQ): This scale is defined by the following formula:

$$
\mathrm{CQ}=\frac{\sum_{\mathrm{i}=1}^{\mathrm{M}} \sum_{\mathrm{j}=1}^{\mathrm{N}} \mathrm{x}(\mathrm{i}, \mathrm{j}) * \mathrm{y}(\mathrm{i}, \mathrm{j})}{\sum_{\mathrm{i}=1}^{\mathrm{M}} \sum_{\mathrm{j}=1}^{\mathrm{N}} \mathrm{x}(\mathrm{i}, \mathrm{j})}
$$

where $x(i, j)$ : Original image element in the location $(i, j)$.

$y(i, j)$ : Optimized image element in the location $(i, j)$.

- Universal Quality Index (UQI): UQI Dynamic range is $[-1,1]$, and at $\left(\mathrm{x}_{\mathbf{i}}=\mathrm{y}_{\mathbf{i}}\right)$ the best value is (1) for all values $\mathbf{i}$ and when $\left(y_{i}=2 \mu_{x}-x_{i}\right)$ lowest value is $(-1)$ for all values $\mathbf{i}$. This model from the list of value sees any contortion is a mix from three elements is diverse is relationship misfortune, bending of luminance, and mutilation of difference. For getting this, the above definition for three parts is revised as:

$$
\mathrm{UQI}=\left(\frac{\sigma_{\mathrm{xy}}}{\sigma_{\mathrm{x}} \sigma_{\mathrm{y}}}\right)\left(\frac{2 \overline{\mathrm{x}} \bar{y}}{\left(\overline{\mathrm{x}}^{2}+\overline{\mathrm{y}}^{2}\right)}\right)\left(\frac{2 \sigma_{\mathrm{x}} \sigma_{\mathrm{y}}}{\left({\sigma_{\mathrm{x}}}^{2}+\sigma_{\mathrm{y}}{ }^{2}\right)}\right)
$$

Where coefficient of correlation is the first component which linear correlation measuring degree between (x, y), and with dynamic range $[-1,1]$. While evaluate components second and third the distortions of relative even if they related linearly. The second component measures how close the mean luminance is between the two images and has range is $[0,1]$.

- Pearson Correlation Coefficient (PCC): This scale is defined by the following formula:

$$
\operatorname{PCC}=\sum_{\mathrm{i}=1}^{\mathrm{M}} \sum_{\mathrm{j}=1}^{\mathrm{N}} \frac{\mathrm{x}(\mathrm{i}, \mathrm{j}) * \mathrm{y}(\mathrm{i}, \mathrm{j})}{\operatorname{Std}[\mathrm{x}(\mathrm{i}, \mathrm{j})] * \operatorname{Std}[\mathrm{y}(\mathrm{i}, \mathrm{j})]}
$$

Std $[x(i, j)]$ : original image element standard deviation at $(i, j)$.

Std $[y(i, j)]$ : optimized image element standard deviation at $(i, j)$.

- Chi-square probability density function (chi2pdf): pdf proceeds of chi-square distribution with freed degrees m, evaluated at the values in $\mathrm{x}$. The chi-square distribution is a one-parameter family of curves. The chi2pdf is:

$$
y=f(x \mid \nu)=\frac{x^{(\nu-2) / 2} e^{-x / 2}}{2^{\frac{\nu}{2}} \Gamma(\nu / 2)}
$$

where $v$ : freedom degrees, $\Gamma(\cdot)$ : Gamma function.

\section{7- RESULTS AND DISCUSSION}

The proposed algorithm which depending on fusion CLAHE enhancement technique is tested on underwater images. Figure (2) shows the underwater images (original) and enhancement images after algorithm (Figure 1) application. The algorithm is designed and implemented using MATLAB software. 
International Journal of Advances in Scientific Research and Engineering (ijasre), Vol 6 (9), September -2020

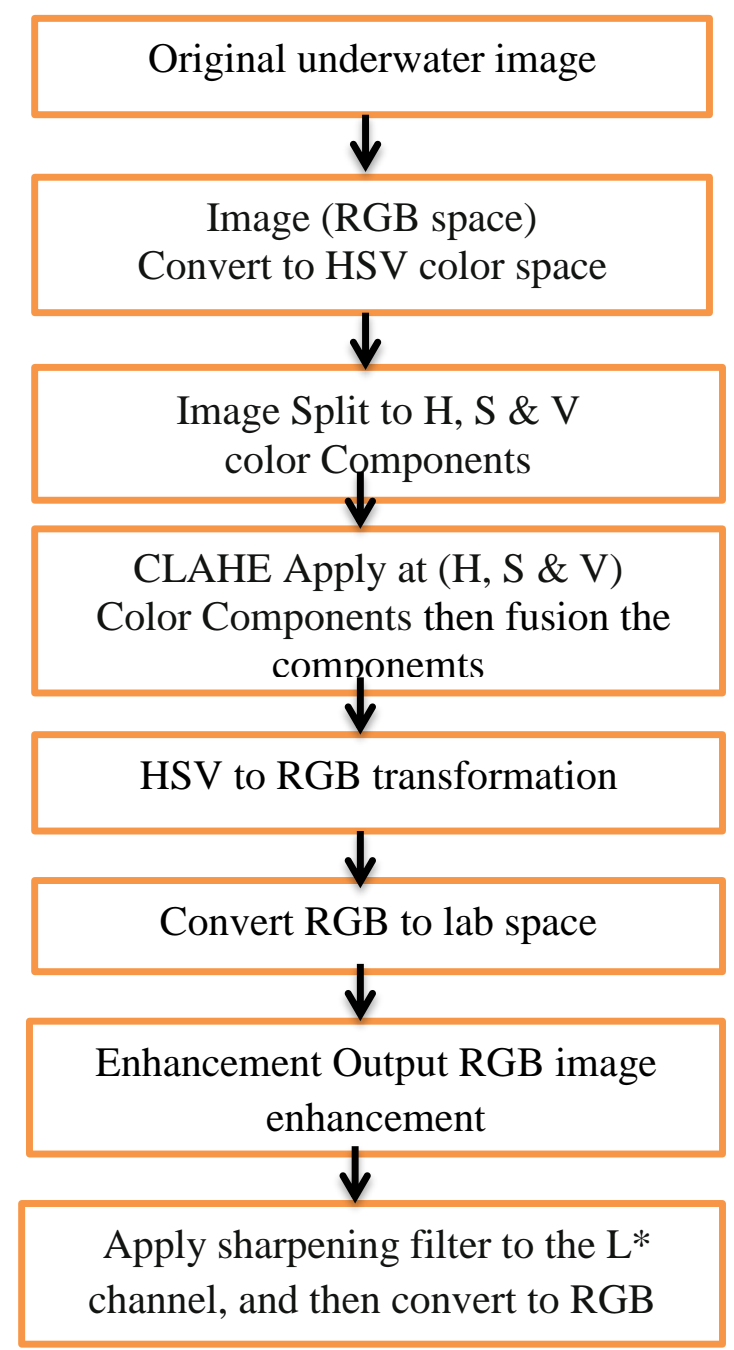

Figure (1) Block diagram of Proposed Algorithm 
International Journal of Advances in Scientific Research and Engineering (ijasre), Vol 6 (9), September -2020

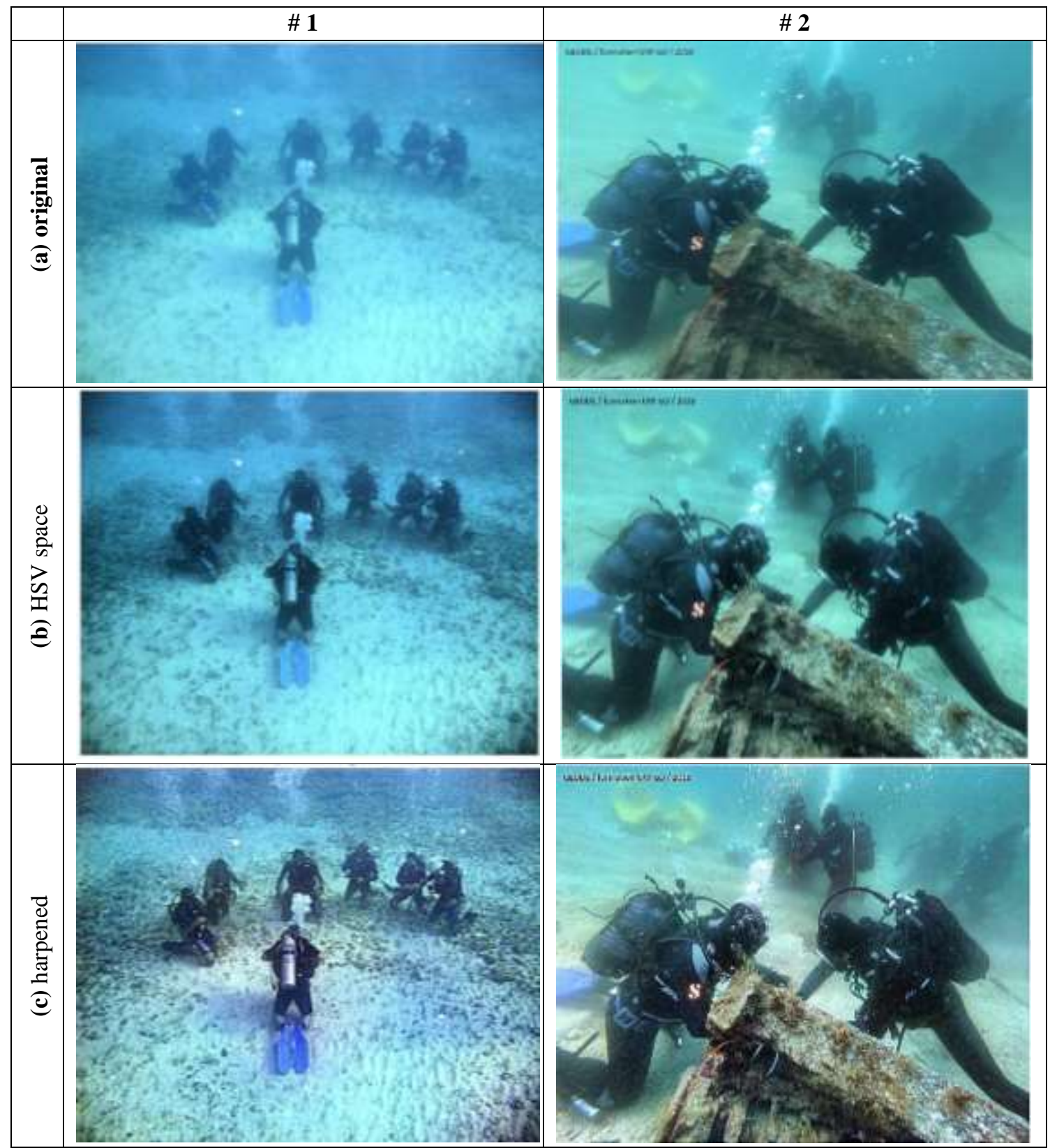

Figure (2) underwater image (a) original

(b) Enhanced image in HSV space (c) sharpened result

Table 1 quantitative metrics of enhancement results for original image figure 2

\begin{tabular}{|c|c|c|l|l|}
\hline Image\# (figure 2) & \multicolumn{2}{|c|}{$\# \mathbf{1}$} & \multicolumn{2}{c|}{ \#2 } \\
\hline Features & HSV space & sharpened & \multicolumn{1}{|c|}{ HSV space } & \multicolumn{1}{|c|}{ sharpened } \\
\hline (RFSIM) & 0.1024 & 0.170 & 0.1024 & 0.170 \\
\hline (RMSE) & 28.99 & 26.70 & 31.45 & 29.34 \\
\hline (PMSE) & 43.42 & 37.10 & 39.78 & 36.14 \\
\hline (LMSE) & 15.19 & 21.34 & 16.21 & 19.78 \\
\hline (PSNR) & 18.88 & 16.31 & 19.64 & 18.38 \\
\hline (MSE) & 8.4 & 10.1 & 8.4 & 10.1 \\
\hline (CQ) & 0.92 & 0.91 & 0.92 & 0.93 \\
\hline (UQI) & 0.9812 & 0.912 & 0.9854 & 0.9741 \\
\hline (PCC) & 0.99 & 0.94 & 0.97 & 0.94 \\
\hline chi2pdf & 0.88 & 0.84 & 0.89 & 0.86 \\
\hline
\end{tabular}

\section{8- CONCLUSION}

An image enhancement technique grounded on histogram equalization is proposed in this study to quality enhance of underwater images. Firstly, CLAHE technique was used for local information enhance in HSV space, and the color image obtained by 
combinations of the three vectors (H, S, and V). Secondly, a sharping filter is used to contrast enhanced of image. The trial results show that the proposed strategy takes care of the issue of shading cast underwater image, lopsided enlightenment, and weak contrast based on better holding the subtleties of the image and upgrades the improvement impact. Compared with the CLAHE improvement technique, and the best results are from comprehensive of the proposed strategy performance, the independent statistical indexes isn't optimal, but subjectively, the color is more distinct and the image details are clearer.

\section{Acknowledgements}

Authors thank Mustansiriya University (uomustansiriyah.edu.iq) Iraq/ Baghdad, for the cooperation extended to them in completing this work.

\section{REFERENCE}

1- C. C. Chiu and C. C. Ting, Contrast enhancement algorithm based on gap adjustment for histogram equalization, Sensors. Vol. 16, p. 936, 2016.

2- Jasneet Kaur Babool, Satbir Singh, Evaluation of single underwater image enhancement with CLAHE, International Journal of Advanced Research in Computer and Communication Engineering, vol. 5, Issue 5, May 2016.

3- Priyanka P. Madhumatke, Pankaj Agarwal, Underwater Image Enhancement Techniques: A Review. International Journal for Research in Applied Science \& Engineering Technology, Vol. 5, Issue VIII, p.51, August 2017.

4- Ghada S. Karam, Ziad M. Abood, Rafal N. Saleh, Enhancement of Underwater Image using Fuzzy Histogram Equalization, International Journal of Applied Information Systems (IJAIS) - ISSN: 2249-0868 Foundation of Computer Science FCS, New York, USA, Vol. 6, No. 6, p.163, December 2013.

5- Ghada Sabah Karam, Fatma Ismail Abbas, Ziad M. Abood, Kadhim K. Kadhim, and Nada S. Karam, An Enhanced Approach for Biomedical Image Restoration Using Image Fusion Techniques, AIP Conference Proceedings, 030028, p. 111, 2018.

7- Shuliang Zou, Qiang LI, Yuhong REN, Dewen Tang, Xin GE, An Effective Fusion Enhancing Approach for Single Underwater Degraded Image, CSSE '19, Xi'an, China. May 24-26, 2019

8- Alex Raj S. M., Deepa S. and Supriya M. H., Underwater Image Enhancement using CLAHE in a Reconfigurable Platform, 978-1-5090-1537- 2016 IEEE.

9- Xi Qiao, Jianhua Bao, Hang Zhang, Lihua Zeng, Daoliang Li, Underwater Image Quality Enhancement of Sea Cucumbers Based on Improved Histogram Equalization And Wavelet Transform. Information Processing in Agriculture, 2017.

10- Mingshi Luo, Yang Fang, Yimeng Ge, An effective underwater image enhancement method based on CLAHE-HF, IOP Conf. Series: Journal of Physics: Conf. Series 1237, 032009, 2019.

11- Marino Mangeruga, Marco Cozza and Fabio Bruno, Evaluation of Underwater Image Enhancement Algorithms under Different Environmental conditions, J. Mar. Sci. Eng., Vol. 6, no. 10, pp. 234-526, 2018.

12- Diksha Garg, Naresh Kumar Garg and Munish Kumar, Underwater image enhancement using blending of CLAHE and percentile methodologies, Multimed Tools Appl., Springer Nature 2018.

13- Zheng L, Shi H, Sun S. underwater image enhancement algorithm based on CLAHE and USM. Proc. IEEE Int. Conf. Inform. Automation (ICIA), pp. 585-590. 2016.

14- Jinxiang Ma, Contrast Limited Adaptive Histogram Equalization-Based Fusion in YIQ and HSI Color Spaces for Underwater Image Enhancement, International Journal of Pattern Recognition and Articial Intelligence Vol. 32, no. 7, p.89, 2018.

15- Ziad M. Abood Ghada S. Karam Rafid. E. Haleot Enhancement Human Face Recognition Based on Fusing of Infrared and Visible Images. Conference 23, College of Education, 26-27 April 2015, Special Issue, no. 2, pp.73-79, 2017.

16- Edgar Chavolla, Daniel Zaldivar, Erik Cuevas and Marco A. Perez, Color Spaces Advantages and Disadvantages in Image Color Clustering Segmentation, Chapter in Studies in Computational Intelligence Springer International Publishing AG Learning in Image Processing, Studies in Computational Intelligence 730, https, pp.3-22, 2018. doi.org/10.1007/978-3-31963754-9_1.

\section{BIOGRAPHIES}

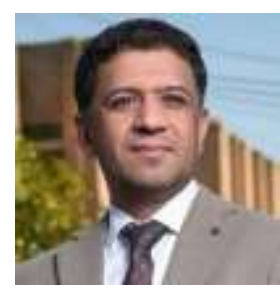

Rafid Aedan Haleot received the bachelor from University of Baghdad, Master's degrees and the Ph.D. degree from from Mustansiriyah University, in 2017. He has nearly 15 years of experience in teaching in computer science department. He is currently a lecturer with the Department of Computer Science, College of Education, Mustansiriyah University. He has published over 8 articles in international and national journals and conferences. His fields of interests include image processing. 